2014

\title{
Two photon absorption laser induced fluorescence measurements of neutral density in a helicon plasma
}

\author{
M.E. Galante \\ R. M. Magee \\ E. E. Scime
}

Follow this and additional works at: https://researchrepository.wvu.edu/faculty_publications

\section{Digital Commons Citation}

Galante, M. E.; Magee, R. M.; and Scime, E. E., "Two photon absorption laser induced fluorescence measurements of neutral density in a helicon plasma" (2014). Faculty Scholarship. 421.

https://researchrepository.wvu.edu/faculty_publications/421 


\title{
Two photon absorption laser induced fluorescence measurements of neutral density in a helicon plasma ${ }^{\text {a) }}$
}

\author{
M. E. Galante, ${ }^{\text {b),c) }}$ R. M. Magee, ${ }^{\text {d) }}$ and E. E. Scime \\ Department of Physics, West Virginia University, Morgantown, West Virginia 26506, USA
}

(Received 22 November 2013; accepted 10 February 2014; published online 30 April 2014)

\begin{abstract}
We have developed a new diagnostic based on two-photon absorption laser induced fluorescence (TALIF). We use a high intensity $\left(5 \mathrm{MW} / \mathrm{cm}^{2}\right)$, narrow bandwidth $\left(0.1 \mathrm{~cm}^{-1}\right)$ laser to probe the ground state of neutral hydrogen, deuterium and krypton with spatial resolution better than $0.2 \mathrm{~cm}$, a time resolution of $10 \mathrm{~ns}$, and a measurement cadence of $20 \mathrm{~Hz}$. Here, we describe proof-of-principle measurements in a helicon plasma source that demonstrate the TALIF diagnostic is capable of measuring neutral densities spanning four orders of magnitude; comparable to the edge neutral gradients predicted in the DIII-D tokamak pedestal. The measurements are performed in hydrogen and deuterium plasmas and absolute calibration is accomplished through TALIF measurements in neutral krypton. The optical configuration employed is confocal, i.e., both light injection and collection are accomplished with a single lens through a single optical port in the vacuum vessel. The wavelength resolution of the diagnostic is sufficient to separate hydrogen and deuterium spectra and we present measurements from mixed hydrogen and deuterium plasmas that demonstrate isotopic abundance measurements are feasible. Time resolved measurements also allow us to explore the evolution of the neutral hydrogen density and temperature and effects of wall recycling. We find that the atomic neutral density grows rapidly at the initiation of the discharge, reaching the steady-state value within $1 \mathrm{~ms}$. Additionally, we find that neutral hydrogen atoms are born with $0.08 \mathrm{eV}$ temperatures, not $2 \mathrm{eV}$ as is typically assumed. (C 2014 AIP Publishing LLC. [http://dx.doi.org/10.1063/1.4873900]
\end{abstract}

\section{INTRODUCTION}

Neutral particles play a key role in all laboratory plasma experiments. Through collisions or photoionization, neutrals are a source of new plasma. Through recombination, chargeexchange collisions, and line radiation, neutrals are a conduit through which plasmas lose energy, momentum, and charged particles. In processing plasmas, neutrals, particularly those in excited electronic states, also play a key role in the chemistry of etching and deposition. In fusion plasmas, neutral gas is introduced through gas puffing at the plasma boundary, through ablation of frozen fuel pellets injected into the core of the plasma, and through recycling of neutrals adsorbed into the walls of the vacuum vessel. Neutral recycling depends on the particular characteristics of the wall material, the temperature of the wall material, the density and temperature of the plasma and gas impinging on the walls, and the exposure history of the wall materials, i.e., was the wall coated with lithium or other gettering materials, was the wall cleaned in a low temperature discharge prior to the primary experiment, and the length of the experiment in pulsed machines. Whereas gas puffing and pellet injection are controlled sources of neutral gas, recycling is particularly problematic. Uncontrolled influxes of neutral gas at the edge of tokamaks can lead to loss of density control, triggering of

\footnotetext{
${ }^{a)}$ Paper JI2 3, Bull. Am. Phys. Soc. 58, 144 (2013).

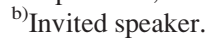

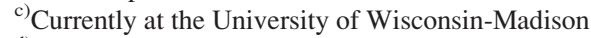

${ }^{d)}$ Currently at Tri Alpha Energy Inc.
}

mode transitions (e.g., from H-mode to L-mode), and ultimately to disruptions. ${ }^{1}$

Many fusion experiments include fast pressure gauges capable of reporting the total gas pressure behind the first wall or in the diverter region. ${ }^{2}$ Such measurements are performed inside the vacuum vessel but outside of the plasma. Direct measurements of the neutral density inside the plasma from the wall to the last closed flux surface are currently provided by inversions of line-integrated emission measurements from excited neutral states, ${ }^{3}$ e.g., $\mathrm{H}-\alpha$ measurements at $656 \mathrm{~nm}$. Determination of the neutral density from such measurements requires sophisticated atomic physics models as well as measurements of electron temperature and electron density throughout the regions of interest. Multiple lines-of-sight, preferably crossing lines-of-sight, are also required for reliable density determination.

Building upon previous experiments in Heliotron and other facilities, ${ }^{4,5}$ we have recently developed a new diagnostic to directly measure the neutral density and neutral temperature in hydrogen, deuterium, and krypton plasma. This diagnostic utilizes two-photon absorption laser induced fluorescence (TALIF) to directly probe the ground state of the neutral particle. ${ }^{6}$ Using this system we have investigated atomic neutral production in hydrogen plasma and neutral dynamics, specifically the effects of neutral depletion, in krypton plasma. Time-resolved measurements in both species reveal a great deal regarding the growth of the atomic neutral density during the formation phase of pulsed hydrogen plasma. The time-resolved measurements of neutral 
temperature of ground state hydrogen atoms indicate that the neutral hydrogen atoms are born with very low temperatures, $0.1 \mathrm{eV}$, not $2.0 \mathrm{eV}$ as is typically assumed.

In Sec. II, we outline the experimental apparatus used in these experiments. TALIF measurements of neutral density and temperature along with discussions of these measurements are presented in Sec. III. In Sec. IV, we summarize the implications of the experimental observations.

\section{HARDWARE}

All measurements presented in this work were obtained in the Compact Helicon for Waves and Instabilities Experiment (CHEWIE), shown in Figure 1. The device is a compact, vertically oriented helicon source that was recently repurposed for diagnostic development. CHEWIE consists of a $60 \mathrm{~cm}$ long, $2.5 \mathrm{~cm}$ radius Pryex ${ }^{\mathrm{TM}}$ glass tube connected to a $20 \mathrm{~cm}$ long, $7.5 \mathrm{~cm}$ radius stainless steel expansion chamber. The top of the glass tube defines $z=0$ and the center of the expansion chamber defines $r=0$. A $m=1$ half-turn right hand helical antenna wraps around the glass tube and is driven with steady-state rf power up to $1 \mathrm{~kW}$ or pulsed power up to $5 \mathrm{~kW}$ at frequencies between $10-16 \mathrm{MHz}$ to create and sustain the plasma. Two 140 turn water-cooled electromagnets in the source region produce a steady-state axial magnetic field of up to $1200 \mathrm{G}$. Nine radial ports in the expansion chamber provide diagnostic access to the plasma. Unless otherwise noted, all experiments presented here were conducted in the expansion region.

The helicon source is operated in three distinct modes: capacitive, inductive, and helicon. Capacitive is a low density mode in which the plasma is created and heated through direct electron acceleration. Inductive is a moderate density

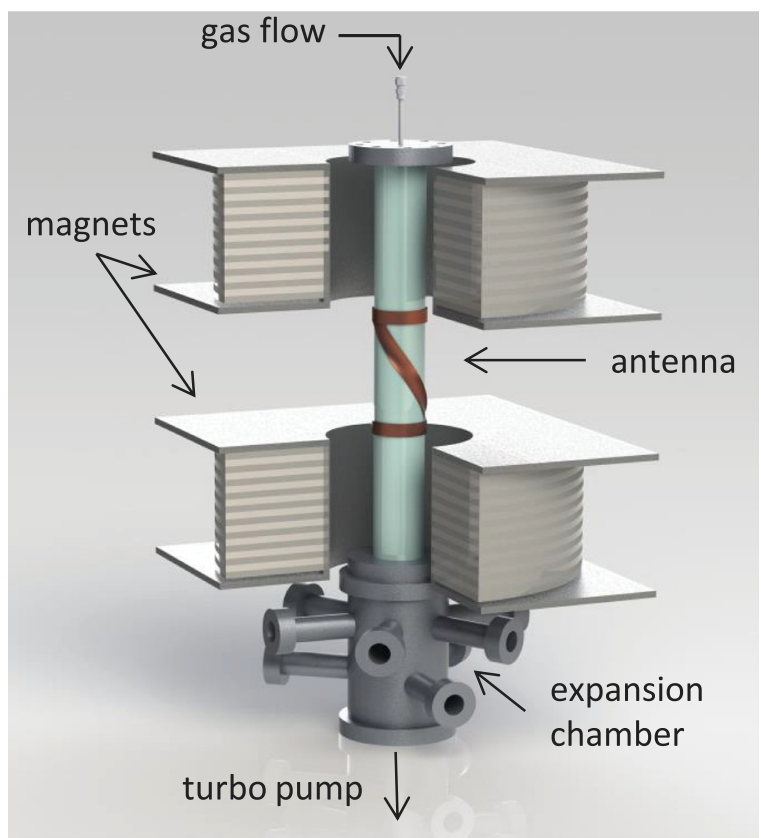

FIG. 1. CHEWIE plasma device. Plasma is created in the glass tube then flows into the expansion chamber below. Unless otherwise noted, the measurements reported here were made in the expansion chamber. Reprinted with permission from Magee et al., Phys. Plasmas 19, 123506 (2012). Copyright 2012 American Institute of Physics. mode in which the plasma is heated and sustained via induction of currents. Helicon is a high density mode in which resonant wave absorption sustains the plasma. The manner in which the wave couples to the plasma is poorly understood and is still an active area of research. ${ }^{7}$ Transitioning between the modes is accomplished through proper tuning of source parameters, i.e., rf power, driving frequency, applied magnetic field strength, etc.

Neutral density measurements were obtained using a new TALIF system. The TALIF system consists of a $20 \mathrm{~Hz}$ pulsed Nd:YAG operating at $532 \mathrm{~nm}$ pumping a scanning dye laser operating around $615 \mathrm{~nm}$. The $615 \mathrm{~nm}$ light from the dye laser is frequency tripled via two non-linear doubling stages producing $8 \mathrm{~ns}$ pulses of up to $8 \mathrm{~mJ}$ at $205 \mathrm{~nm}$. A complete discussion of the system can be found elsewhere. ${ }^{6}$ As shown in Figure 2, simultaneous absorption of two $205.14 \mathrm{~nm}$ photons excites the $1 s \rightarrow 3 d$ transition in neutral hydrogen (deuterium). Radiative decay from the $3 d \rightarrow 2 p$ results in the emission of a $656.4 \mathrm{~nm}$ photon. The system is absolutely calibrated in a 1-10 mTorr fill of cold krypton gas. ${ }^{8}$ Details regarding calibration, including the krypton TALIF scheme, are provided in Ref. 6.

TALIF overcomes many of the issues associated with other neutral density diagnostics. Specifically, and most importantly, because each photon contributes a portion of the energy needed for the target transition, it is possible to probe high energy transitions from the ground state; enabling direct measurement of the ground state density and temperature of neutral atoms. Directly probing the ground state eliminates the need for complicated numerical models needed to calculate the ground state density from LIF measurements of excited states. ${ }^{9,10}$ Such models also require measurements of the plasma density and electron temperature in the regions of interest.

The line width of the laser is spectrally narrow enough, $\sim 0.1 \mathrm{pm}$, that hydrogen and deuterium neutral absorption lines are easily resolved in a low temperature, mixed gas discharge, see Figure 3. The narrow line width permits realtime measurement of isotopic abundance, an important figure of merit in fusion plasmas where proper fuel mix is required to optimize fusion gain.

The TALIF system is operated in a confocal scheme, shown in Figure 4, in which light is injected and collected through a single porthole. As depicted in Figure 4 laser light is directed through a UV grade fused silica lens to a roughly

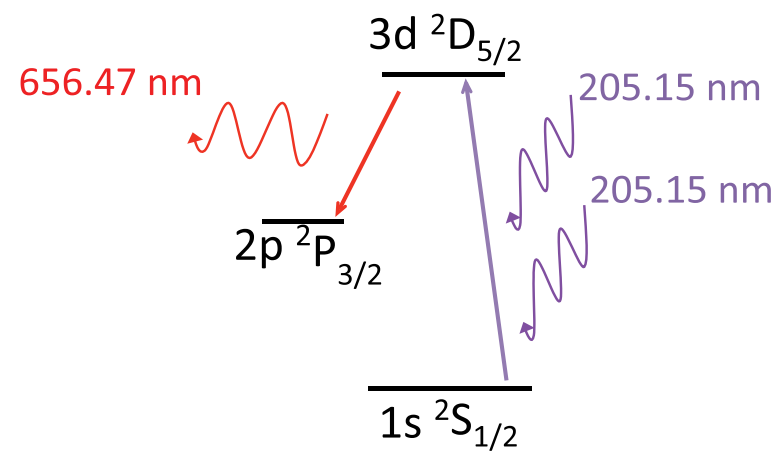

FIG. 2. Hydrogen TALIF scheme used in these experiments. 


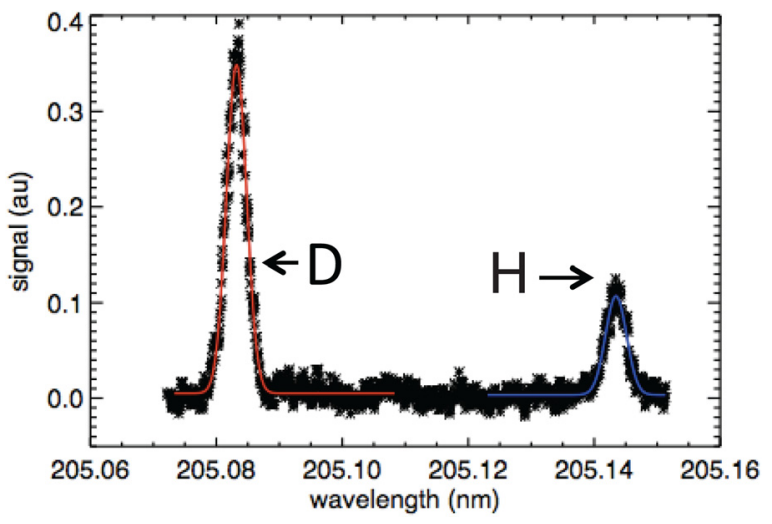

FIG. 3. Spectrally resolved deuterium and hydrogen neutrals in a mixed species discharge.

$60 \mu \mathrm{m}$ diameter spot in the plasma. Due to the highly localized nature of TALIF (the TALIF signal is proportional to the square of the laser intensity), fluorescence is only emitted from a small region around the focal spot. A portion of the fluorescence is emitted back along the line of injection, is collimated by the focusing lens and is then directed with a $45^{\circ}$ turning mirror into a filtered high-speed photomultiplier tube (PMT). To reject background light at other wavelengths, a bandpass filter centered on $656 \mathrm{~nm}$ is placed in front of the PMT. A $10 \mathrm{~mm}$ diameter hole in the $5 \mathrm{~cm}$ turning mirror allows the injected beam to pass and reduces the collection area by $4 \%$. The hole in the mirror also prevents all background light along the optical axis from entering the PMT. The focusing lens is mounted on a translation stage with spatial resolution better than $1 \mathrm{~mm}$. Moving the lens moves the location of the focal spot in the plasma and enables measurement of radial profiles through the single viewport. All measurements presented here were performed at laser intensities below the saturation intensities for krypton and hydrogen. ${ }^{6}$

\section{EXPERIMENTAL MEASUREMENTS}

\section{A. Neutral profiles in krypton}

The phenomenon of neutral depletion has been observed in a number of helicon devices for a number of different species. ${ }^{9-11}$ Neutral pumping, the mechanism suggested for neutral depletion beyond that expected due to ionization, generally follows two scenarios. In the first, neutral particles

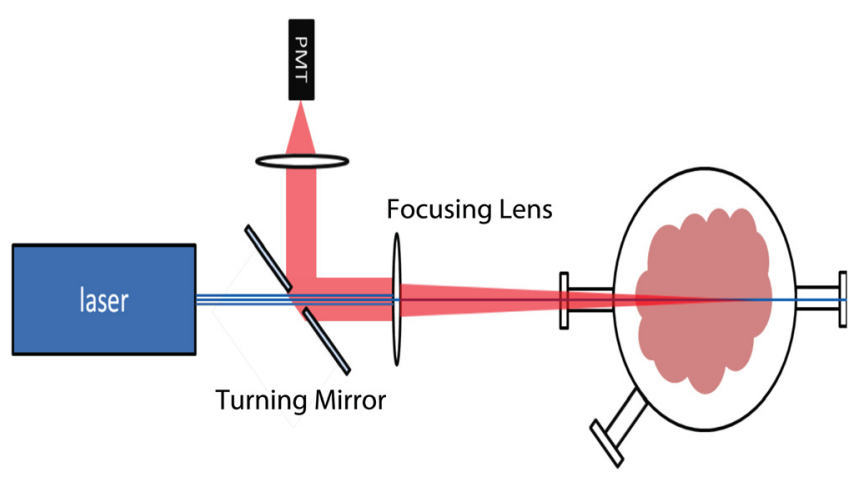

FIG. 4. Confocal injection/collection scheme. See text for description. enter the plasma and are ionized through collisions with electrons. These fresh ions are then accelerated out of the plasma to the chamber wall at the sound speed by a sheath electric field. In the low density edge, the ions recombine and new neutrals drifts back into the plasma at the neutral thermal speed. The large difference between ion sound speed and neutral thermal speed results in a net loss of ions from the core. In the second mechanism, pressure balance between the hot plasma and cold neutrals results in neutral expulsion from the plasma core. ${ }^{12}$ Whatever the mechanism, the result is the same, a decrease in the core neutral density beyond that expected solely from ionization.

For certain operating parameters (rf power, magnetic field strength, driving frequency) significant neutral depletion is observed in krypton plasma. Such neutral depletion is only observed when the source is in the helicon operating mode, see Figure 5. In inductive mode the majority of the $\mathrm{rf}$ power is deposited at the edge of the plasma and the neutral and plasma density profiles are relatively flat. When the helicon mode is achieved, either by increasing of power, magnetic field strength, or decreasing the driving frequency, the power is deposited in the center of the plasma column. The result is a centrally peaked plasma density profile and a very hollow neutral density profile. However, the neutral density decreases more than the observed increase in plasma density, thus there is a mechanism beyond ionization forcing neutrals out of the core. ${ }^{13}$

Axial measurements reveal significant neutral depletion in the source region. As shown in Figure 6, with increasing rf power the neutral density in the antenna region, denoted by the shaded region, decreases dramatically. Above $900 \mathrm{~W}$, the density is below the diagnostic's resolution. Plasma density measurements are unavailable in this region. However,
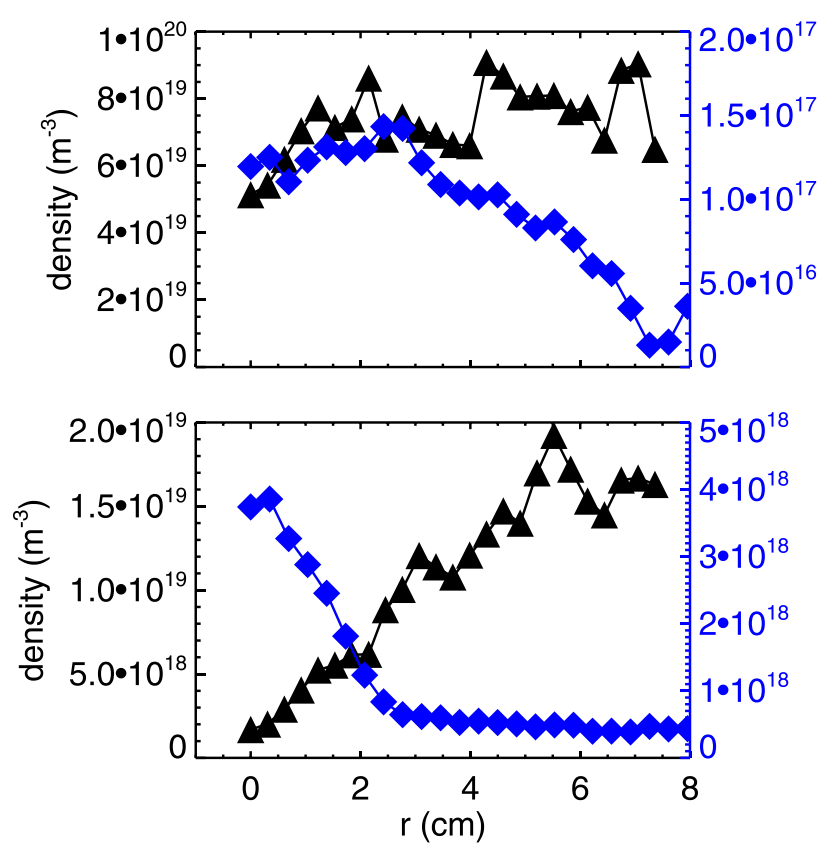

FIG. 5. Krypton plasma (diamonds) and neutral (triangles) density as a function of radius in the inductive (top) and helicon (bottom) operating modes. Reprinted with permission from Magee et al., Phys. Plasmas 19, 123506 (2012). Copyright 2012 American Institute of Physics. 


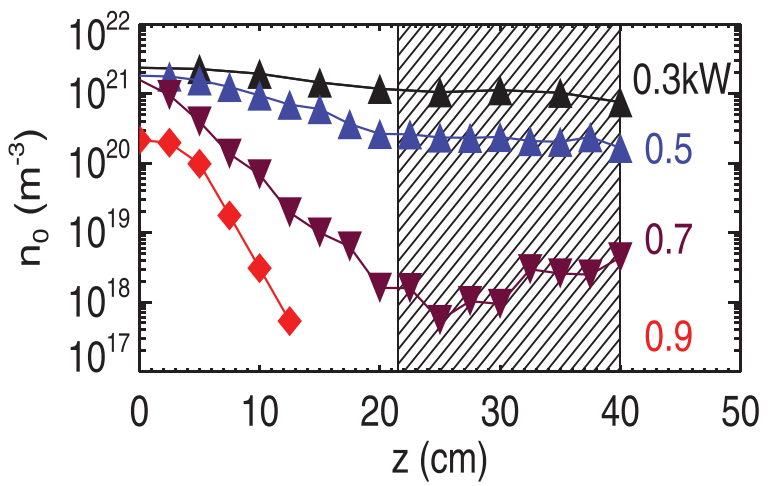

FIG. 6. Axial neutral krypton density as a function of rf power. Shaded section is the antenna location, with $z=0$ the top of the glass tube. The neutral density continues to drop with increasing power despite the constant plasma density above $1 \mathrm{~kW}$. The growth region of empty neutrals is consistent with the plasma increasing in axial extent, forcing more neutrals out of the core. Reprinted with permission from Magee et al., Phys. Plasmas 20, 123511 (2013). Copyright 2013 American Institute of Physics.

plasma density measurements in the expansion region, shown in Figure 7, reveal that the plasma density plateaus at $1 \%$ of the fill density at $1 \mathrm{~kW}$ of rf power and is then constant despite increasing the rf power to $5 \mathrm{~kW}$. Thus, the density plateau is not due to insufficient rf power. This suggests that neutral depletion in the source region is limiting the achievable plasma density in the expansion region. The fully ionized source effectively starves the downstream region, preventing the increase in plasma density there even with the factor of 5 increase in $\mathrm{rf}$ power. ${ }^{14}$

\section{B. Neutral profiles in hydrogen}

While the krypton neutral density changed dramatically in both magnitude and profile shape with changing operating parameters, no such changes were observed in hydrogen plasmas. Flat neutral density profiles were measured for all source parameters. The lack of significant profile changes suggests that the addition of the molecular species in some way alters the power absorption mechanism in the plasma. Specifically, the addition of molecular dissociation to the energy balance provide a significant pathway for absorbing rf power without creating plasma, resulting in the much

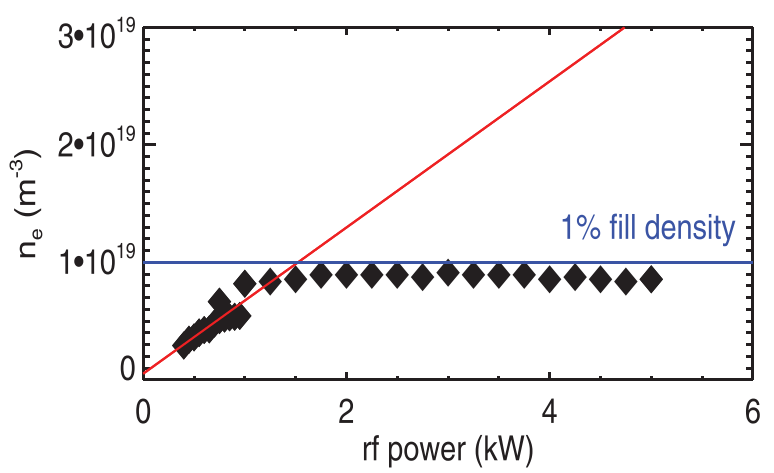

FIG. 7. Plasma density as a function of rf power in the expansion region. Above a critical power, $\sim 1 \mathrm{~kW}$, the plasma density stops increasing despite reaching a density only $1 \%$ of the fill density. The plateau in the plasma density suggests that the plasma is starved of neutrals, preventing further increases in density. ${ }^{14}$ lower levels of ionization for all operating parameters in hydrogen compared to krypton.

The shape of the hydrogen profile suggests the influence of a fast neutral transport mechanism that results in a flat density profile. Neutral hydrogen production primarily arises from collisions of molecular hydrogen with free electrons. Thus, the neutral hydrogen profile should reflect the shape of the electron density profile. However, as shown in Figure 8, the atomic neutral profile is flat even when the plasma density becomes highly centrally peaked. Either atomic neutrals are not purely the result of electron-molecule collisions, highly unlikely, or the rate of neutral transport out of the center is so large that the neutral profile remains flat. The profile flattening could be explained by neutral diffusion occurring faster than neutral creation by dissociating collisions. Time resolved measurements in the source region show rapid neutral growth with the neutral density reaching its steady state value within $1 \mathrm{~ms}$. For the atomic neutral temperatures in these experiments, $<0.1 \mathrm{eV}$, the atomic neutral flow can be described as molecular, i.e., $\lambda_{m f p} \gg l_{d}$, where $\lambda_{m f p}$ is the mean free path for atomic-molecular collisions, and $l_{d}$ is some characteristic length of the plasma chamber. In this case the atomic neutral confinement time is ${ }^{15}$

$$
\tau=\frac{l_{d}}{v_{t h}} .
$$

For a $0.1 \mathrm{eV}$ atomic neutral and a characteristic chamber length of $7.5 \mathrm{~cm}$ the atomic confinement time is $\sim 20 \mu \mathrm{s}$. Thus, neutrals will diffuse out of the center of the discharge faster than they are created, relaxing the profile shape much faster than it can steepen.
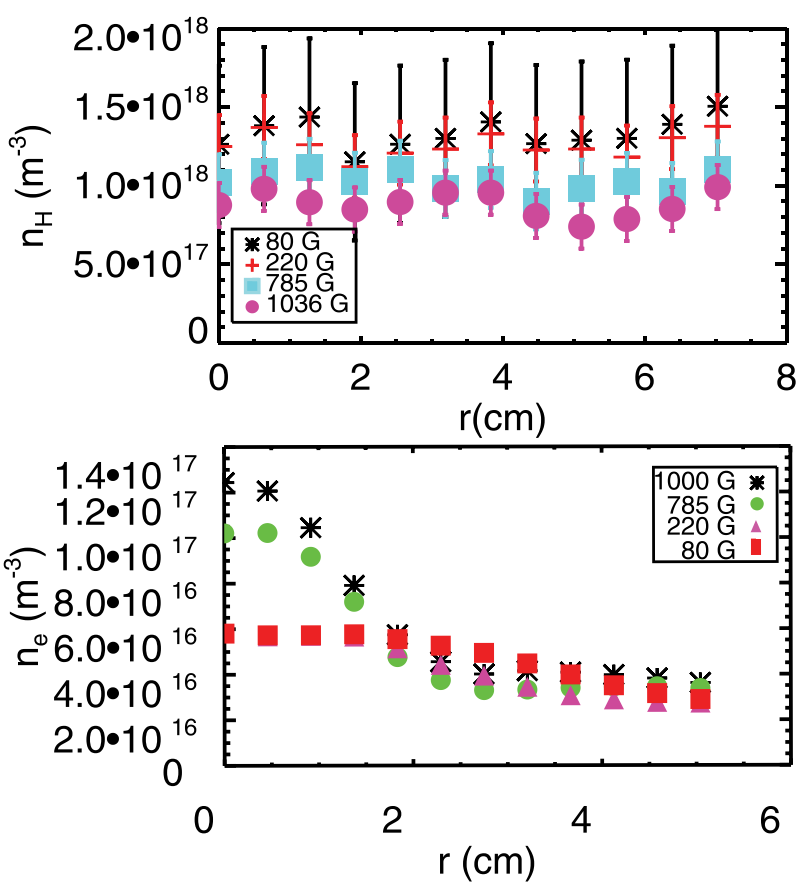

FIG. 8. Atomic hydrogen (top) and plasma (bottom) density for a range of magnetic field strengths. At high field, above $785 \mathrm{G}$, the plasma density profile becomes centrally peaked but the neutral density profile stays flat. 


\section{Isotopic differences}

Similar behavior with changing operating parameters is observed between hydrogen and deuterium plasma. However, as is shown in Figure 9, the deuterium neutral and plasma densities are approximately a factor of 2 larger than the hydrogen neutral and plasma densities for identical source parameters. The factor of 2 increase is suggestive of a mass factor resulting in higher levels of both dissociation and ionization.

The difference in plasma and atomic neutral densities in hydrogen and deuterium plasma could again be explained by neutral diffusion. Deuterium, having a larger mass, will have a longer diffusion time than hydrogen for the same neutral temperature. Because of the increase in diffusion time, deuterium neutrals spend more time in the higher density region of the plasma, resulting in more opportunities to be ionized, or dissociated. The result is a larger plasma and atomic neutral density than in the lighter hydrogen gas. Additional studies involving molecular gasses with increasing mass are needed to verify this hypothesis.

\section{Time resolved neutral density and temperature}

In addition to directly measuring the ground state neutral density, ground state neutral temperatures were also directly measured. As shown in Figure 10, the neutral temperature was low in steady state plasmas, $\sim 0.08 \mathrm{eV}$, and the neutral temperature profile was flat.

In an atomic species such a temperature might be expected, as there is no mechanism heating the neutrals. However, in a molecular plasma, where atomic neutrals are the result of dissociating collisions, it might be expected that the neutrals are born with finite energy resulting from the dissociation process. In hydrogen it is typically expected that atomic neutrals are Franck-Condon neutrals. These are neutrals born with $\sim 2 \mathrm{eV}$ energies through the reaction

$$
e+H_{2} \rightarrow e+H_{2}^{*} \rightarrow e+H(1 s)+H(1 s),
$$

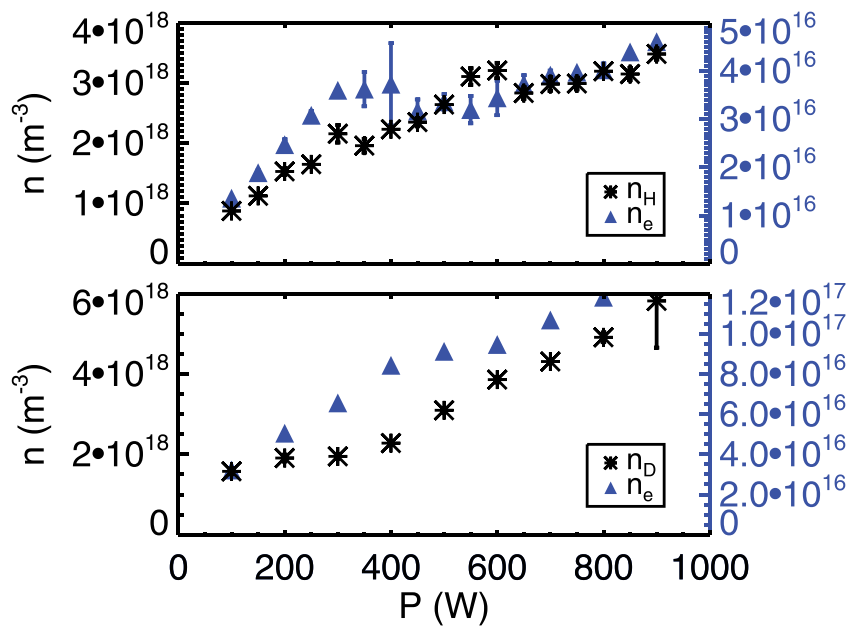

FIG. 9. Atomic hydrogen (top) and deuterium (bottom) density as a function of rf power. Deuterium neutral and plasma densities were larger than hydrogen for all source parameters. Error bars for most of the data points are smaller than the symbols used.

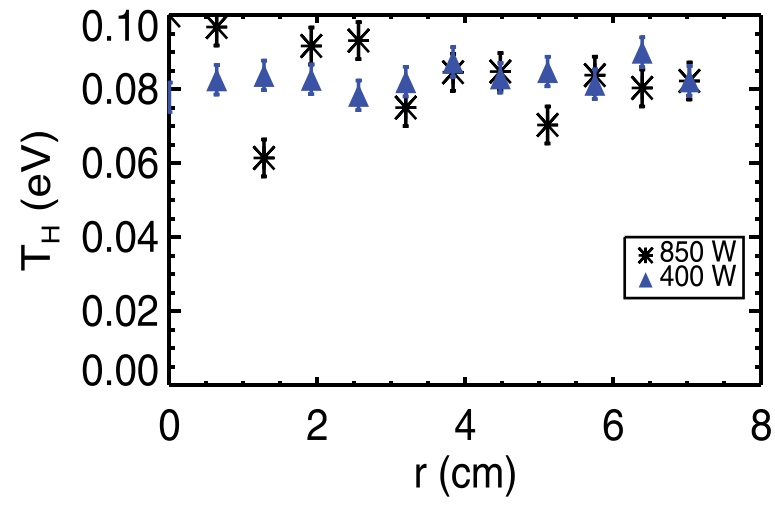

FIG. 10. Neutral hydrogen temperatures for two different rf powers.

where $H_{2}^{*}$ is an excited molecule. ${ }^{16}$ Most codes simulating the edge of magnetic fusion devices usually treat all neutrals as Franck-Condon neutrals. ${ }^{17}$ These measurements suggest that the assumption of a Franck-Condon neutral birth process may not be warranted in laboratory plasmas.

It is possible that neutrals are born at high energy then equilibrate with the chamber walls to the measured temperature. However, as shown in Figures 11 and 12, while the atomic neutral density ramps in $\sim 1 \mathrm{~ms}$ there is no change in the atomic neutral temperature. The atomic neutrals are born with $\sim 0.08 \mathrm{eV}$ energies, the same energy as is measured in steady-state. It is possible that there are multiple energy neutral populations. However, as is shown in Figure 13, the measured neutral hydrogen spectrum is well described by a single Maxwellian. Thus, there is no evidence for the existence of a two temperature neutral distribution, i.e., a high density population with low temperature and a low density population at a higher, $\sim 2 \mathrm{eV}$, temperature.

\section{E. Wall loading}

In addition to fast time-resolved measurements, TALIF measurements made on a very long (hours) timescale, reveal a significant, $2-3 x$, growth in the neutral density in both hydrogen and deuterium, see Figure 14. The growth was measured following extended, $8 \mathrm{~h}$, krypton discharges. This behavior is consistent with wall loading in the expansion chamber. The krypton discharges effectively "clean" the walls in the expansion chamber. The walls are heated, forcing them to outgas. Such a technique is routinely used with

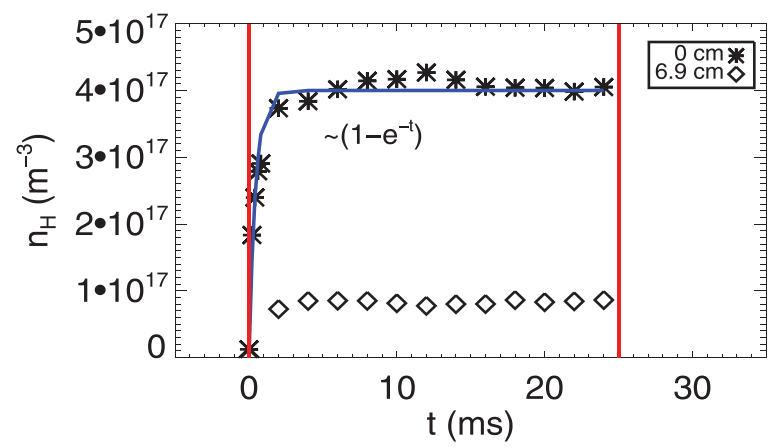

FIG. 11. Neutral hydrogen density as a function of time in the center (stars) and edge (diamonds) of the expansion chamber. Vertical lines indicate the start and end of the discharge. 


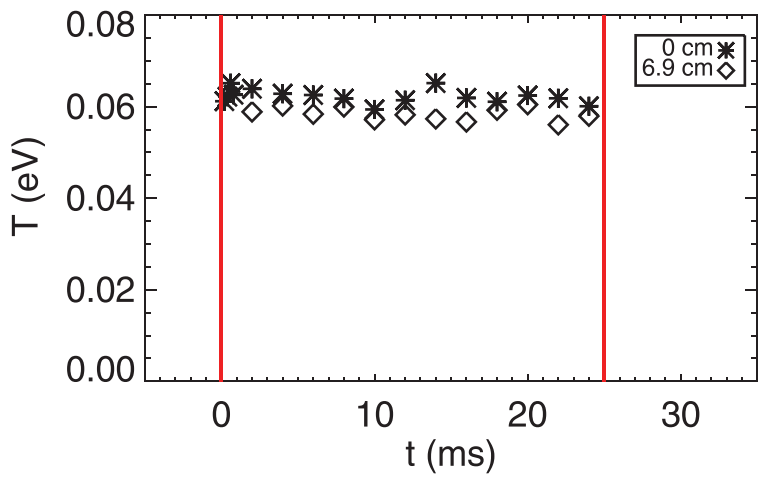

FIG. 12. Neutral hydrogen temperature as a function of time in the center (stars) and edge (diamonds) of the expansion chamber. Vertical lines indicate the start and end of the discharge.

helium in magnetic fusion devices. ${ }^{18}$ When hydrogen plasmas are created following krypton, hydrogen neutrals are adsorbed into the "empty" stainless steel up to a saturation point. As the walls approach saturation fewer neutrals adhere to the walls, increasing the density of neutrals in the plasma. The decrease in adsorption is consistent with an increase in neutral density for longer discharge times. The difference in wall loading saturation level and rate of wall loading between hydrogen and deuterium is consistent with the difference in density between the species. More deuterium neutrals in the plasma result in a larger fluence of deuterium atoms to the wall, resulting in faster wall loading. Thus, it is not surprising that the rate of wall loading is faster in deuterium. Wall loading studies such as these are very important for fusion devices, in which wall retention and subsequent release can affect plasma performance. Understanding how neutrals are retained and released from plasma facing walls provides insight into the recycling process.

\section{CONCLUSIONS}

A new, absolutely calibrated, neutral density diagnostic has been constructed and demonstrated. The diagnostic is based on two-photon absorption laser induced fluorescence

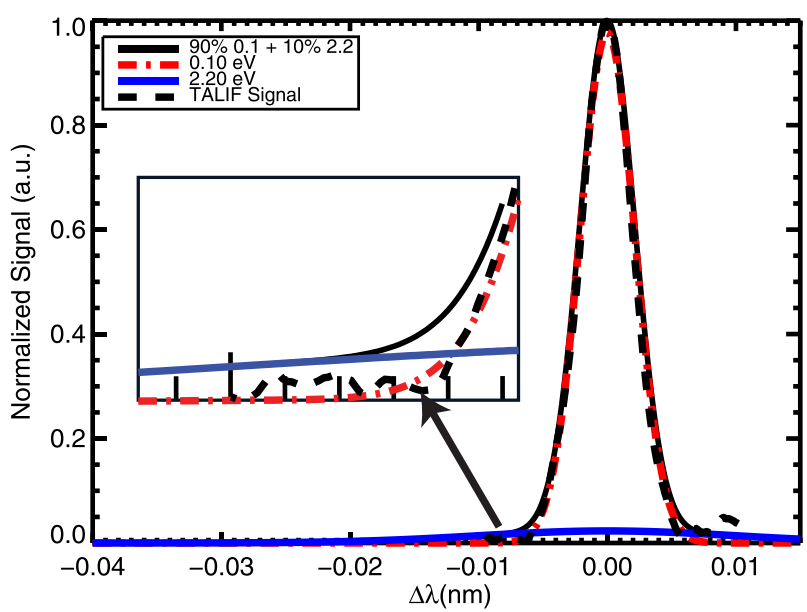

FIG. 13. Distribution for two temperature populations. Dashed line is the fit to an experimental measurement. Inset shows zoomed in view of the wing of the distribution. The experimental data (dashed line) in the wings are well fit by the single low temperature population (dot-dashed line), not the two temperature distribution (solid black line).

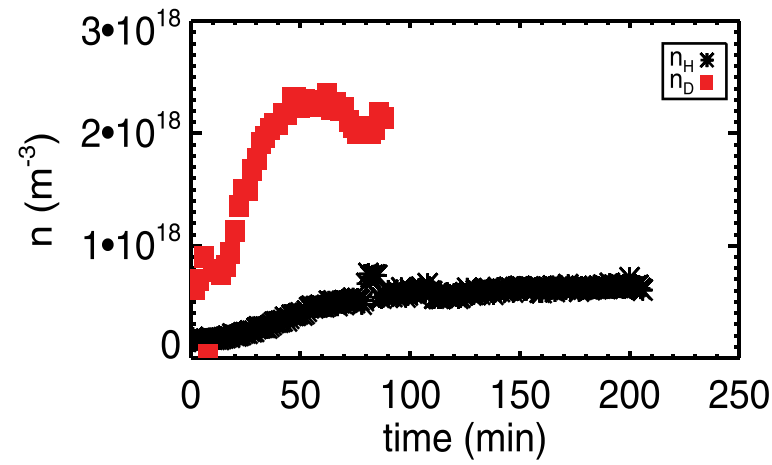

FIG. 14. Neutral hydrogen (stars) and deuterium (squares) as a function of discharge time. Ramp in density suggests wall loading on a long (minutes) time scale.

and was used to directly investigate the neutral density in krypton, hydrogen and deuterium plasma. The diagnostic provides a direct measure of the ground state density, eliminating the need for complex modeling to calculate ground state quantities in low temperature and fusion plasmas. Significant neutral depletion is observed in krypton plasma when operating in helicon mode, consistent with previous excited state LIF measurements in argon and helium plasma. Neutral depletion sets an effective density limit on the plasma and results in the source approaching $100 \%$ ionization fraction in the core despite a maximum krypton plasma density of only $1 \%$ of edge measured fill density. No neutral depletion was observed in hydrogen, presumably due to the low levels of ionization achieved. The difference in ionization levels between hydrogen (deuterium) and krypton suggests the addition of molecular dissociation to the energy balance provides a significant avenue for power absorption preventing achievement of similar plasma densities. Low steady-state neutral temperatures were measured for all source parameters and time-resolved measurements suggest that neutrals are born at low energy, contrary to what is conventionally expected. Lastly, long time scale neutral growth consistent with wall loading was observed in both hydrogen and deuterium plasmas.

\section{ACKNOWLEDGMENTS}

Special thanks to D. McCarren and J. A. Reusch for useful discussions. Work supported by U.S. DOE Grant No. DE-SC0004736.

${ }^{1}$ V. Mertens, M. Kaufmann, J. Neuhauser, J. Schweinzer, J. Stober, K. Büchl, O. Gruber, G. Haas, A. Herrmann, A. Kallenbach et al., Nucl. Fusion 37, 1607 (1997).

${ }^{2}$ A. Niemczewski, I. H. Hutchinson, B. LaBombard, B. Lipschultz, and G. M. McCracken, Nucl. Fusion 37, 151 (1997).

${ }^{3}$ R. L. Boivin, J. A. Goetz, A. E. Hubbard, J. W. Hughes, I. H. Hutchinson, J. H. Irby, B. LaBombard, E. S. Marmar, D. Mossessian, C. S. Pitcher et al., Phys. Plasmas 7, 1919 (2000).

${ }^{4}$ T. Kajiwara, K. Takeda, K. Muraoka, T. Okada, M. Maeda, and M. Akazaki, Jpn. J. Appl. Phys. 29, L826 (1990).

${ }^{5}$ H. F. Döbele, U. Czarnetzki, and A. Goehlich, Plasma Sources Sci. Technol. 9, 477 (2000).

${ }^{6}$ R. M. Magee, M. E. Galante, D. McCarren, E. E. Scime, R. L. Boivin, N. H. Brooks, R. J. Groebner, D. N. Hill, and G. D. Porter, Rev. Sci. Instrum. 83, 10D701 (2012). 
${ }^{7}$ A. R. Ellingboe and R. W. Boswell, Phys. Plasmas 3, 2797 (1996).

${ }^{8}$ K. Niemi, V. S. von der Gathen, and H. F. Döbele, J. Phys. D: Appl. Phys. 34, 2330 (2001).

${ }^{9}$ A. M. Keesee and E. E. Scime, Plasma Sources Sci. Technol. 16, 742 (2007).

${ }^{10}$ S. Houshmandyar and E. E. Scime, Plasma Sources Sci. Technol. 21, 035008 (2012).

${ }^{11}$ A. Aanesland, L. Liard, G. Leray, J. Jolly, and P. Chabert, Appl. Phys. Lett. 91, 121502 (2007).

${ }^{12}$ A. Fruchtman, G. Makrinich, P. Chabert, and J. M. Rax, Phys. Rev. Lett. 95, 115002 (2005).
${ }^{13}$ R. M. Magee, M. E. Galante, N. Gulbrandsen, D. W. McCarren, and E. E. Scime, Phys. Plasmas 19, 123506 (2012).

${ }^{14}$ R. M. Magee, M. E. Galante, J. Carr, Jr., G. Lusk, D. W. McCarren, and E. E. Scime, Phys. Plasmas 20, 123511 (2013).

${ }^{15}$ U. Fantz, Technical Report IPP 10/21, Max-Planck-Institut für Plasmaphysik, 2002.

${ }^{16}$ R. K. Janev, W. D. Langer, K. Evans, and D. E. Post, Elementary Processes in Hydrogen-Helium Plasmas (Springer-Verlag, 1987).

${ }^{17}$ R. J. Groebner, M. A. Mahdavi, A. W. Leonard, T. H. Osborne, G. D. Porter, R. J. Colchin, and L. W. Owen, Phys. Plasmas 9, 2134 (2002).

${ }^{18}$ J. Ehrenberg, J. Nucl. Mater. 162-164, 63 (1989). 